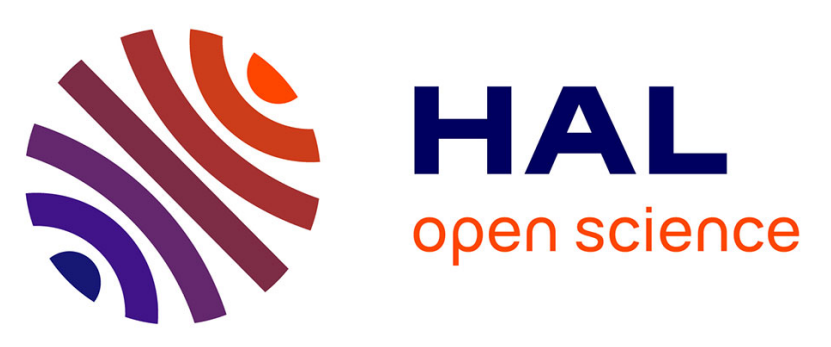

\title{
Resveratrol formulated with a natural deep eutectic solvent inhibits active matrix metalloprotease-9 in hormetic conditions.
}

Aly Shamseddin, Céline Crauste, Erwann Durand, Pierre Villeneuve, Gregor Dubois, Thierry Durand, Joseph Vercauteren, Francisco Veas

\section{To cite this version:}

Aly Shamseddin, Céline Crauste, Erwann Durand, Pierre Villeneuve, Gregor Dubois, et al.. Resveratrol formulated with a natural deep eutectic solvent inhibits active matrix metalloprotease-9 in hormetic conditions.. European Journal of Lipid Science and Technology, 2017, pp.1700171. 10.1002/ejlt.201700171 . hal-02308915

\section{HAL Id: hal-02308915 https://hal.science/hal-02308915}

Submitted on 8 Oct 2019

HAL is a multi-disciplinary open access archive for the deposit and dissemination of scientific research documents, whether they are published or not. The documents may come from teaching and research institutions in France or abroad, or from public or private research centers.
L'archive ouverte pluridisciplinaire HAL, est destinée au dépôt et à la diffusion de documents scientifiques de niveau recherche, publiés ou non, émanant des établissements d'enseignement et de recherche français ou étrangers, des laboratoires publics ou privés. 


\title{
Research Article
}

\section{Resveratrol formulated with a natural deep eutectic solvent inhibits active matrix metalloprotease- 9 in hormetic conditions}

\author{
Aly Shamseddin ${ }^{1}$, Céline Crauste ${ }^{2}$, Erwann Durand ${ }^{3}$, Pierre Villeneuve ${ }^{3}$, Gregor Dubois ${ }^{1}$, \\ Thierry Durand ${ }^{2}$, Joseph Vercauteren ${ }^{2}$ and Francisco Veas ${ }^{1}$ \\ ${ }^{1}$ Molecular Comparative Immuno-Physiopathology Lab (LIPMC), French Research Institute for Development \\ (IRD), UMR-Ministry of Defense, Faculty of Pharmacy, Montpellier University, Montpellier, France \\ 2 Institute for Biomolecules Max Mousseron (IBMM), UMR 5247 CNRS-UM-ENSCM, Faculty of Pharmacy, \\ Laboratory of Pharmacognosy, Montpellier University, Montpellier, France \\ ${ }^{3}$ International French Center for Agronomy Research (CIRAD), UMR-IATE, Montpellier, France
}

Despite the promising anti-oxidant and anti-inflammatory effects of resveratrol (RES) on human health, pre-clinical and clinical studies are frequently disappointing, probably due to its low water-solubility and poor bioavailability. Even though a hormetic mode of action was clearly established for RES, the high doses commonly used to mitigate these issues, lead to adverse effects. Common hallmarks of multiple pathologies results from pathological-enhanced endothelial permeability due to both enhanced inflammation and matrix metalloprotease-9 (aMMP-9) activities. The main aim of this work was to optimize the RES capacity to inhibit aMMP-9 by using a new class of solvents, natural deep eutectic solvents (NADES) for a new RES formulation as compared with dimethyl-sulfoxide (DMSO). To obtain the appropriate NADES, 18 compounds combinations were prepared to select those exhibiting the optimized capacity to dissolve RES. The RES-NADES 1,2-propanediol:choline-chloride:water (PCW, 1:1:1 molar ratio) and compared with RES-DMSO for their aMMP-9-inhibitory capacities. Low concentrations of RES-NADES/PCW formulation exhibited both a biocompatible solubility and a strong increased aMMP-9-inhibitory activity, at least 10-fold, higher than RES-DMSO, reaching its hormetic mode of action. Following in vivo validations, some particular NADES could potentially be considered as the new generation of formulation for druggable compounds.

Practical applications: Formulation of resveratrol in Natural Deep Eutectic solvents (NADES) optimizes its capacity to inhibit active matrix metalloprotease-9. The Resveratrol-NADES 1,2-propanediol:choline-was the most efficient and low concentrations exhibited both a biocompatible solubility and an increased aMMP-9-inhibitory activity, at least 10-fold, higher than RES-DMSO. Consequently, the NADES/PCW formulation allowed resveratrol to reach its hormetic mode of action. Following in vivo validations, some particular NADES could potentially be considered as the new generation of formulation for druggable compounds.

Keywords: Hormesis / MMP-9 / Metalloproteases / nADES (natural deep eutectic solvents) / Resveratrol

Received: April 10, 2017 / Revised: June 28, 2017 / Accepted: July 17, 2017

DOI: $10.1002 /$ ejlt.201700171

Supporting information available online https://doi.org/10.1002/ejlt.201700171

Correspondence: Francisco Veas, Molecular Comparative ImmunoPhysiopathology Lab (LIPMC), French Research Institute for Development (IRD), UMR-Ministry of Defense, Joseph Vercauteren, Institute for Biomolecules Max Mousseron (IBMM), UMR 5247 CNRS-UM-ENSCM, Laboratory of Pharmacognosy, Faculty of Pharmacy, Montpellier University, Montpellier 34093, France

E-mail: francisco.veas@ird.fr and jvercauteren@univ-montp1.fr Fax: +33411759546

Abbreviations: aMMP, active matrix metalloproteinase; BxPC3, human pancreatic adenocarcinoma cell line; hcMec/D3, human brain microvascular endothelial primary-derived cell-line; HUVEC, human umbilical vein endothelial cells; MDA MB-231, human breast adenocarcinoma cell-line; MMP, matrix metalloproteinase; NADES, natural deep eutectic solvent; NADES/PCW, NADES 1,2-propanediol choline chloride:water; RES, resveratrol; THP-1, human monocyte cell line; TNF- $\alpha$, tumor necrosis factor-alpha

\section{Introduction}

Numerous clinical studies have been performed with resveratrol (3, $4^{\prime}, 5$-trihydroxystilbene, RES), a plant polyphenol from the stilbenoid series (Fig. 1), to assess its capacity to promote beneficial effects on human health $[1,2]$. Indeed RES, is considered as a dietary anti-oxidant [3], able to decrease inflammatory processes, including the inhibition of the gelatinolytic metalloproteases (gMMP)-2 and 9 activities [4]. The activity increase of gMMP is strongly related to pathological enhancement of endothelial

Additional corresponding author: Joseph Vercauteren. E-mail: joseph.vercauteren@umontpellier.fr 


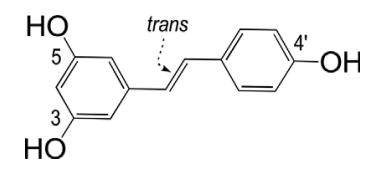

Figure 1. Resveratrol structure and numbering of transresveratrol.

permeability is a common characteristic of several infectious and systemic diseases, including HIV-1 [5], dengue virus infection [6], Hantavirus cardiopulmonary syndrome [7], and cancer [8]. There are multiple MMP-9 upstream enhancers, such as pro-inflammatory cytokines (tumor necrosis factor- $\alpha, \mathrm{TNF}-\alpha)$, growth and transcriptional factors [9]. Despite the previous mentioned positive effects of RES in human health, its low-water solubility, mode of action, targets, route and dose of administration, as well as its low bioavailability, preclude its regular use as a therapeutic approach [10-13]. In order to overcome the poor watersolubility of RES, and to be able to observe measurable effects, some groups, using elevated RES doses (1000 to $1500 \mathrm{mg} /$ person/day), did not get the positive effects observed in humans at low concentrations $(150 \mathrm{mg} /$ day $)$ as reported by others [14, 15] but, rather observed adverse effects $[16,17]$. For more than 10 years, such a phenomenon has been assigned to hormetic effect of RES [18-23]. Some synonymous terms of hormesis have also been used, such as biphasic dose-response effect, adaptive response, or preconditioning effects $[18,24]$. All these data advocate for the use of the lowest effective RES doses, in preclinical and/or clinical studies. Different formulations have been set up to reach the goal to solve both the RES solubility and bioavailability issues: Micellar solutions with bile acids [25], carboxymethyl chitosan (CMCS) and RES-loaded CMCS nanoparticles (RES-CMCSNPs) [26], $N$-trimethyl chitosan (TMC) grafted by palmitic acid (TMC-g-PA) [27], cyclodextrin, and co-encapsulation of cyclodextrin inclusions in liposomes [28]. Some of these nanotechnology-based formulations were able to successfully "enhance the RES clinical potential" [29].

For the first time in this field, this study is using a new class of solvents so called Natural Deep Eutectic Solvents (NADES), recently discovered by Choi et al. [30], to prepare new formulations of RES. NADES consist in a mixture, in specific molar ratios, of two or more plant-based metabolites, exhibiting strong capacity of self-association through noncovalent interactions, ionic interactions, hydrogen bonding, and van der Waals forces (lipophilic-lipophilic and $\pi-\pi$ interactions), leading to a supramolecular assembly. The molecules forming such structures stay connected to each other through a well-organized three-dimensional system, acting as a new entity with properties mostly different from those of pure isolated components (melting point, solving power,...). One of the most important changes is the drastic lowering of melting point: NADES act like a pure compound (eutectic) and melt at very low temperature (deep). Unlike the very similar noncovalent derivatives such as ionic liquids (IL) and deep eutectic solvents (DES), the main components of NADES are all natural: Sugars, amino acids, and organic acids. NADES are very "good solvents" due to the strong intermolecular interactions between NADES components, They are used in broad-spectrum applications, including cosmetics, media for enzymatic reactions [31, 32], enhancers for extraction of bioactive natural products, and nucleic acids [33, 34]. In addition, NADES have been shown to be good solvents for non-water-soluble small molecules (polyphenols), or even, macromolecules, (starch, cellulose, nucleic acids [33]).

From 18 compound combinations were used to prepare different NADES, we selected those exhibiting the highest RES solubilizing power and were used to formulate RES (RES-NADES). The best combination used as a RES formulation was compared with a RES DMSO-formulation (RES-DMSO), which is the most current solvent of medium polar substrates for in vitro studies. For the first time here, we show that RES was not only easily dissolved in a particular NADES, but also that this formulation exhibited at least a 10-fold higher aMMP-9 inhibitory effect as compared with the RES-DMSO formulation, making possible to lower RES doses and thus able to reach the hormetic effects conditions.

\section{Materials and methods}

\subsection{Chemicals and reagents}

Dimethyl sulfoxide (DMSO) and MTT (3-[4,5-Dimethylthiazol-2-yl]-2,5-diphenyltetrazolium bromide; Thiazolyl blue), as well as individual NADES components were purchased from Sigma-Aldrich ${ }^{\mathrm{TM}}$ (Marnes-la-Coquette, France), Choline chloride $(\mathrm{ChCl})$ was dried under vacuum at $60^{\circ} \mathrm{C}$ over a silica gel for $72 \mathrm{~h}$ before use. TNF- $\alpha$ was purchased from PeproTech ${ }^{\mathrm{TM}}$ (Paris, France), and all other chemicals used in this study were highly purified molecular grade reagents (Sigma, Paris, France). RES was isolated from stalks of Vitis vinifera, Vitaceae, and highly-purified (98\%) according to the process described by Delaunay et al. [35].

\subsection{Preparation of natural deep eutectic solvents}

A set of 18 natural deep eutectic solvents were prepared according to the procedure modified from Abbott [36]. Briefly, these NADES were prepared as follows: Components 1, 2 and 3 , from (Table 1), were directly weighed in a flask, avoiding any contact with the air moisture, to get a molar ratio as described in (Table 1). Finally, all mixtures to form a potential NADES were heated and stirred with an orbital shaker at $250 \mathrm{rpm}$ and incubated at $60^{\circ} \mathrm{C}$ until a clear solution was formed. Among these solvents, three solvents were able to solubilize RES (as visually assessed), NADES composed of: 1,2-propanediol: choline-chloride:water $(1: 1: 1)$ (NADES/PCW), Glucose: 
Table 1. Components of tested natural deep eutectic solvents

\begin{tabular}{lcccc}
\hline NADES & Compound 1 & $\begin{array}{c}\text { Compound } \\
2\end{array}$ & $\begin{array}{c}\text { Compound } \\
3\end{array}$ & $\begin{array}{c}\text { Molar } \\
\text { ratio }\end{array}$ \\
\hline 1 & Glycerol & ChCl & - & $2: 1$ \\
2 & Lactic acid & Betaine & - & $2: 1$ \\
3 & Lactic acid & ChCl & - & $10: 1$ \\
4 & Malic acid & ChCl & - & $1: 1$ \\
5 & Malic acid & Glycine & - & $1: 1$ \\
6 & Malic acid & Proline & - & $1: 3$ \\
7 & Malonic acid & ChCl & - & $1: 1$ \\
8 & Urea & ChCl & - & $2: 1$ \\
9 & 1,2-propanediol & ChCl & Water & $1: 1: 1$ \\
10 & Glucose & ChCl & Water & $1: 2.5: 2.5$ \\
11 & Glycerol & ChCl & Water & $2: 1: 2$ \\
12 & Glycerol & Proline & Sucrose & $9: 4: 1$ \\
13 & Lactic acid & Glucose & Water & $5: 1: 1$ \\
14 & Lactic acid & Proline & Water & $1: 1: 0.25$ \\
15 & Malic acid & Proline & Water & $1: 1: 3$ \\
16 & Sucrose & ChCl & water & $1: 1: 1$ \\
17 & Sucrose & ChCl & Water & $1: 4: 4$ \\
18 & Urea & ChCl & Water & $2: 1: 2$ \\
\hline
\end{tabular}

ChCl:Water (1:2.5:2.5) (NADES/GCW), and Malic acid: $\mathrm{ChCl}$ (1:1) (NADES/MA). NADES/PCW was further selected based upon both its higher RES solubilizing capacity as well as its individual low cytotoxicity.

\subsection{Determination of resveratrol solubility in NADES/PCW and DMSO}

To assess the RES concentration by UPLC-UV analysis (Accela-Thermo scientific), a calibration curve was realized using standard samples of RES, diluted in water to respectively get final concentrations of $16,8,4,2$, and $1 \mu \mathrm{g} / \mathrm{mL}$. The UPLC-UV analysis of RES was performed using an Xbridge BEH C18 2.5 $\mu \mathrm{m}$ column $(2.1 \times 100 \mathrm{~mm})$ and an Xbridge BEH C18 $2.5 \mu \mathrm{m}$ pre-column $(2.1 \times 5 \mathrm{~mm})$. Solvents $\mathrm{A}\left(\mathrm{H}_{2} \mathrm{O}\right)$ and $\mathrm{B}\left(\mathrm{CH}_{3} \mathrm{CN}\right)$ were used according to the timetable in the following gradient: $80 / 20$ from 0 to $3 \mathrm{~min}, 80 / 20$ to $50 / 50$ from 3 to $10 \mathrm{~min}, 50 / 50$ to $80 / 20$ from 10 to $11 \mathrm{~min}$, all of them at a flow rate of $0.3 \mathrm{~mL} / \mathrm{min}$. The RES detection was performed at $310 \mathrm{~nm}$. In order to determine the experimental working solubility of RES in both solvents NADES and DMSO, $20 \mathrm{mg}$ of RES powder were dissolved in either $1 \mathrm{~mL}$ of NADES/PCW or $1 \mathrm{~mL}$ of DMSO and heated overnight at $40^{\circ} \mathrm{C}$. Subsequently, these preparations were centrifuged for $5 \mathrm{~min}$ at $1500 \mathrm{rpm}$ and supernatants were recovered. To prepare a theoretical RES concentration of $1 \mathrm{mg} / \mathrm{mL}, 10 \mu \mathrm{L}$ of the respective recovered supernatant were diluted with $190 \mu \mathrm{L}$ of methanol, from which a theoretical $10 \mu \mathrm{g} / \mathrm{mL}$ solution was realized in $\mathrm{H}_{2} \mathrm{O}\left(10 \mu \mathrm{L}\right.$ in $\left.990 \mu \mathrm{L} \mathrm{H} \mathrm{H}_{2} \mathrm{O}\right)$. All these solutions were analyzed by the UPLC-UV method (using the calibration curve) allowed us to determine the actual concentration in each sample and to compare the corresponding RES solubility in both NADES/PCW and DMSO (supplementary Fig. S1).

\subsection{Cell culture}

A $\mathrm{TNF}-\alpha$ activated human leukemia cell line (THP-1) was used as a source of active MMP-9 production. In the aim to assess the MMP-9-inhibitory activity of RES, RES was dissolved in two kinds of solvents, NADES/PCW and DMSO. The cytotoxic activity of different compounds was assessed using the MTT assay on different cell types, including primary human umbilical vein endothelial cells (HUVEC), human leukemia cell line (THP-1), the human brain micro-vascular endothelial cell line (hcMEC/D3) was derived from primary human temporal lobe micro-vessels (immortalized with a lentiviral vector transduction with the catalytic subunit of human telomerase (hTERT) and SV40 large $\mathrm{T}$ antigen [37] as well as other cell lines including the human pancreatic adenocarcinoma $\mathrm{BxPC} 3$, and the human breast adenocarcinoma MDA MB-231. Both THP-1 and BxPC3 were cultured in RPMI 1640, whereas MDA MB-231 was cultured in DMEM medium, and both media were supplemented with $10 \%$ heat-inactivated FBS, penicillin $\mathrm{G} 100 \mathrm{U} / \mathrm{mL}$ and streptomycin $100 \mu \mathrm{g} / \mathrm{mL}$ purchased from Fisher Scientific ${ }^{\mathrm{TM}}$ (Illkirch-Graffenstaden, France). HUVEC were maintained in EndoGro ${ }^{\mathrm{TM}}$ low-serum culture media kit purchased from Merck Millipore ${ }^{\mathrm{TM}}$ (Paris, France) and hcMEC/D3 were maintained in EBM-2 $\mathrm{mL} \mathrm{V}^{\mathrm{TM}}$ culture media kit purchased from Lonza (Basel, Switzerland).

\subsection{Inhibition of the MMP-9 gelatinolytic activity}

In the presence of TNF- $\alpha 10 \mathrm{ng} / \mathrm{mL}, \mathrm{THP}-1$ monocytes were seeded at $3 \times 10^{5}$ cell $/ \mathrm{mL}$ in 24 -well plate and were incubated with different concentrations of $\operatorname{RES}(1,2,4,8,10,20$, and $30 \mu \mathrm{M})$ dissolved either in DMSO or NADES/PCW. Different THP-1 controls were prepared by incubating cells with each solvent in RPMI cell culture medium at a final concentration of $0.2 \% \mathrm{DMSO}$ or $0.2 \% \mathrm{NADES} / \mathrm{PCW}$, in the presence or in the absence of TNF- $\alpha$. After $24 \mathrm{~h}$ of cell culture in these conditions, $1 \mathrm{~mL}$ of supernatants were collected and centrifuged at $1200 \mathrm{rpm}$ at $25^{\circ} \mathrm{C}$ for $5 \mathrm{~min}$ before their storage at $-80^{\circ} \mathrm{C}$ up to the zymogram analysis.

\subsection{Zymography}

The gelatinolytic activity of MMP-9 was assessed using a zymography approach as described by Pan et al. [38]. Briefly, supernatants were collected as mentioned above and thawed to be electrophoretically separated in a $10 \%$ SDS-polyacrylamide gel electrophoresis (PAGE) containing 1\% gelatin in the absence of reducing agents. After the electrophoresis, gels were washed three times in a solution of $2.5 \%$ Triton $\mathrm{X}-100$, followed by incubation with a gelatinase buffer $(\mathrm{NaCl}$ 
$200 \mathrm{mM}$, Tris Base $50 \mathrm{mM}, \mathrm{CaCl}_{2} 5 \mathrm{mM}$, and $\mathrm{ZnCl}_{2}$ $0.25 \mathrm{mM} ; \mathrm{pH} 7.5)$ on an orbital shaker at $100 \mathrm{rpm}$ at $37^{\circ} \mathrm{C}$ for $24 \mathrm{~h}$. Then, gels were stained using the Coomassie Blue-staining solution ( $0.025 \%$ Coomassie Blue, $40 \%$ methanol, and $10 \%$ acetic acid) for $1 \mathrm{~h}$, followed by treatment of distaining solution (glacial acetic acid 10\% and methanol $20 \%$ ), until white bands are formed surrounded by the blue background. Gel bands were photographed and analyzed using GelAnalyzer $2010 \mathrm{a}^{\mathrm{TM}}$ software.

\subsection{MTT cytotoxicity assay}

The MTT cytotoxicity assay relies on the reading intensity of the converted formazan blue crystals from tetrazolium dye by living cell, the followed protocol was modified of the protocol recommended by Mosmann [39]. THP-1, BxPC3, MDA-MB 231 , and hcMEC/D 3 cells were seeded at $5 \times 10^{3}$ cells/well in 96-well plate for $24 \mathrm{~h}$. While HUVEC were seeded at $1 \times 10^{4}$ cells/well density in the same conditions. Then, supernatants were discarded and plates were either treated with different concentrations of NADES/PCW, NADES/GCW, NADES/ MA or DMSO $(0.5,1$, and $2 \%)$, or with serial dilutions $(10,20$, 40 , and $80 \mu \mathrm{M}$ ) of each RES formulation (RES in NADES/ PCW or RES in DMSO). Samples were assayed in duplicates for each concentration, and normalized against negative control of each solvent diluted in the corresponding cell culture medium ie., 0.2\% NADES (PCW, GCW, MA)treated or $0.2 \%$ DMSO-treated. Subsequently, plates were incubated for additional $72 \mathrm{~h}$ in a $\mathrm{CO}_{2}$ humid chamber at $37^{\circ} \mathrm{C}$ before replacing the medium by MTT in a serum-free medium and incubated for four additional hours. To dissolve formazan crystals, SDS-HCl 10\% was added to each plate and incubated at $37^{\circ} \mathrm{C}$ for $2 \mathrm{~h}$, the optical density was measured at $570 \mathrm{~nm}$ (against the reference, $690 \mathrm{~nm}$ ) using a TECAN $^{\mathrm{TM}}$ plate reader (Paris, France).

\subsection{Statistic analyses}

Experimental data from each group were analyzed using GraphPad Prism ${ }^{\text {TM }}$ v. 5 software. To analyze the differences between NADES/PCW and DMSO treated groups, onepaired Student $t$-test was carried out. All experiments were done least three times with replicates equal or higher than two. The same test was also applied for the cytotoxicity assay between different groups. Values of $p \leq 0.05$ were considered statistically significant.

\section{Results}

\subsection{Toxicity assay of NADES (PCW, GCW, and MA) and DMSO}

Some particular NADES including PCW, GCW, and MA, preselected for their capacity to solubilize RES (by visualization), were assessed for their cytotoxic effects on either THP-1 (Fig. 2A) monocytes or primary HUVEC (Fig. 2B), using the MTT assay. The results showed that at a $2 \%$ NADES/PCW concentration exhibited high deleterious impact on respective THP-1 and HUVEC viability of 71 and $85 \%$, respectively. DMSO decreased the viability of THP-1 and HUVEC to 60 and $80 \%$, respectively. When NADES/ PCW or DMSO was assayed at the lower concentration of $0.5 \%$, no toxicity was observed neither against THP-1, nor HUVEC. NADES/GCW exhibited high cell toxicity at $2 \%$ that rapidly declined in function of a decreased concentration. In contrast, NADES/MA was extremely toxic at all the tested concentrations for both THP-1 and HUVEC. Since, NADES/PCW and DMSO exhibited the best solubility (supplementary material, Fig. S1) and the lowest cytotoxicity of NADES/PCW (Fig. 2C) and DMSO (Fig. 2D) was extended to other cell types, including $\mathrm{BxPC} 3$, hcMec/D3 and MDA MB-231. The highest cytotoxicity effect of NADES/PCW and DMSO was observed at $2 \%$ that remained relatively moderated, since the cell observed viability varied from 60 to $81 \%$ in these cells (Table 2). In contrast, the cytotoxicity was drastically reduced at the lowest assayed concentration of $0.5 \%$.

\subsection{Resveratrol cytotoxicity}

To properly assess the cytotoxicity of RES, the final used concentration of solvents, either NADES/PCW or DMSO to prepare RES was $0.2 \%$, at least fivefold reduced concentration as compared with their cytotoxic effect at a concentration of $1 \%$ (Fig. 2). These results show that high concentrations ( 40 and $80 \mu \mathrm{M}$ ) of RES have a similar high cytotoxicity level for both THP-1 monocytes (Fig. 3A) and primary HUVEC (Fig. 3B). In addition, as shown in Table 3, the $50 \%$ of lethal concentration $\left(\mathrm{LC}_{50}\right)$ of both RES formulations for THP-1 and HUVEC were relatively high, as established using GraphPad ${ }^{\mathrm{TM}}$ from Prism v.5 software. Indeed, both $\mathrm{LC}_{50}$ values of RES-NADES/PCW and RESDMSO tested with THP-1 monocytes were close to $40 \mu \mathrm{M}$. While the LC $_{50}$ of RES-NADES/PCW and RES-DMSO tested with primary HUVEC were similar, respectively 60 and $64 \mu \mathrm{M}$. In contrast, for the lower concentrations NADES/PCW and DMSO allowed high cell viability as compared to the negative control cells submitted to RES vehicles.

\subsection{Resveratrol formulated with NADES/PCW or DMSO and the MMP-9 activity}

The MMP-9-inhibitory activity of RES dissolved in $0.2 \%$ NADES/PCW or $0.2 \%$ DMSO was assessed on a culture of TNF- $\alpha$-activated THP-1 monocytes, which shed large amounts of active MMP-9 into the supernatant. Zymography results (Fig. 4A) clearly showed a MMP-9-inhibitory activity of RES. The pixel density analyses of each gel band 

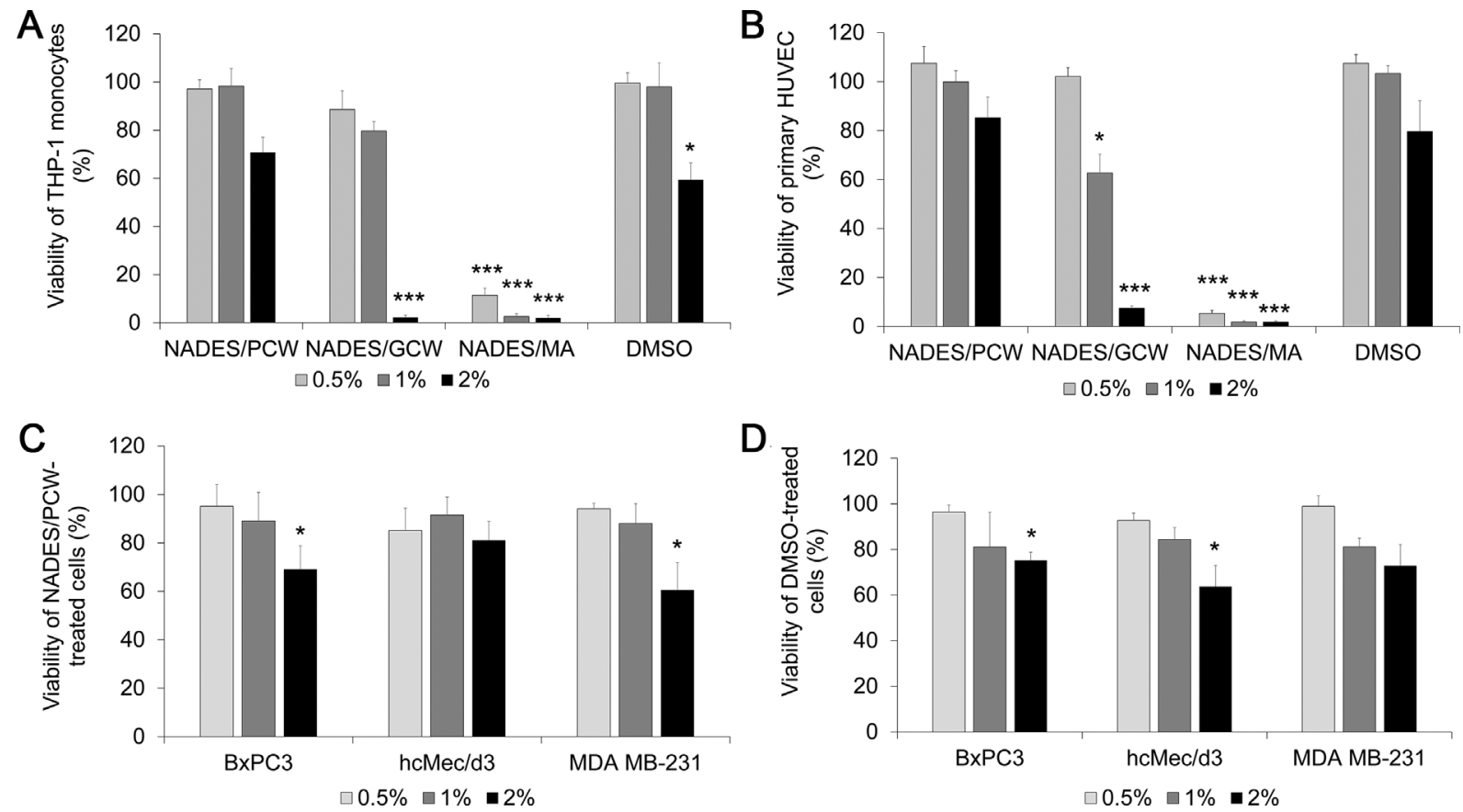

Figure 2. In vitro cytotoxic effects of different RES solvents: NADES (PCW, GCW, and MA) and DMSO. Increasing concentrations $0.5,1$, and $2 \%$ of NADES/PCW, NADES/GCW, NADES/MA, or DMSO were incubated with either THP-1 monocytes (A) or primary HUVEC (B) and then submitted to MTT cytotoxicity assay. Subsequently, in the same conditions these data were extended to other three different cell types including BxPC3, hcMec/D3, and MDA MB-231 for solvent having previously shown the lowest cytotoxic effects NADES/PCW (C) and DMSO (D). Values are mean of three experiments \pm standard deviation. ${ }^{*} p \leq 0.05$, and ${ }^{* * *} p \leq 0.001$.

representing the MMP-9 activity confirmed a decrease of this activity in function of the inhibitory activity of RES. These data were plotted for each concentration of both RES formulations (Fig. 4B). We must stress that a significant increase of the RES-NADES/PCW-MMP-9 inhibitory activity was observed at low RES concentrations up to $1 \mu \mathrm{M}$ that was more than 10 -fold stronger than the limited inhibitory activity of RES-DMSO. Thus, evidencing that the NADES/PCW vehicle was able to confirm and enhance the hormetic mode of action of RES as compared with its limited dose-response activity when dissolved in DMSO.

\section{Discussion}

Our findings revealed that the selected natural deep eutectic solvent 1,2-Propanediol 1,2: ChCl:water or NADES/PCW
(1:1:1) was able to dissolve stalks-extracted RES native pure powder. The UPLC-UV quantification analyses have evidenced that RES was completely soluble in NADES/PCW at a RES-saturated concentration of $20 \mathrm{~g} / \mathrm{L}$ at $40^{\circ} \mathrm{C}$, and that this solubility was similar than the one measured in the RES-DMSO formulation (supporting information file, Fig. S1). Since the NADES/PCW has exhibited both the highest visual solubilizing power and lower cytotoxicity of RES as compared with the two other selected RES solvents NADES/GCW and NADES/MA (Fig. 2), these latter were discarded and only NADES/PCW was used to assess the capacity of RES to inhibit the active form of MMP-9 produced from TNF- $\alpha$-activated THP-1 monocytes. The individual toxicity of NADES/PCW, DMSO, or resveratrol was assessed using the MTT assay. Thus, we observed that both NADES/PCW and DMSO, in the absence of RES were both toxic at the concentration of $2 \%$, as shown in (Table 2 ).

Table 2. Cell viability assay of either NADES/PCW or DMSO in the absence of RES

\begin{tabular}{lccccc}
\hline & \multicolumn{5}{c}{ Cells viability (\%) } \\
\cline { 2 - 6 } Solvent 2\% & HUVEC & THP-1 & hcMec/D3 & BxPC3 & MDA MB-231 \\
\hline NADES/PCW & 85 & 71 & 81 & 69 & 75 \\
DMSO & 80 & 60 & 63 & 73 \\
\hline
\end{tabular}



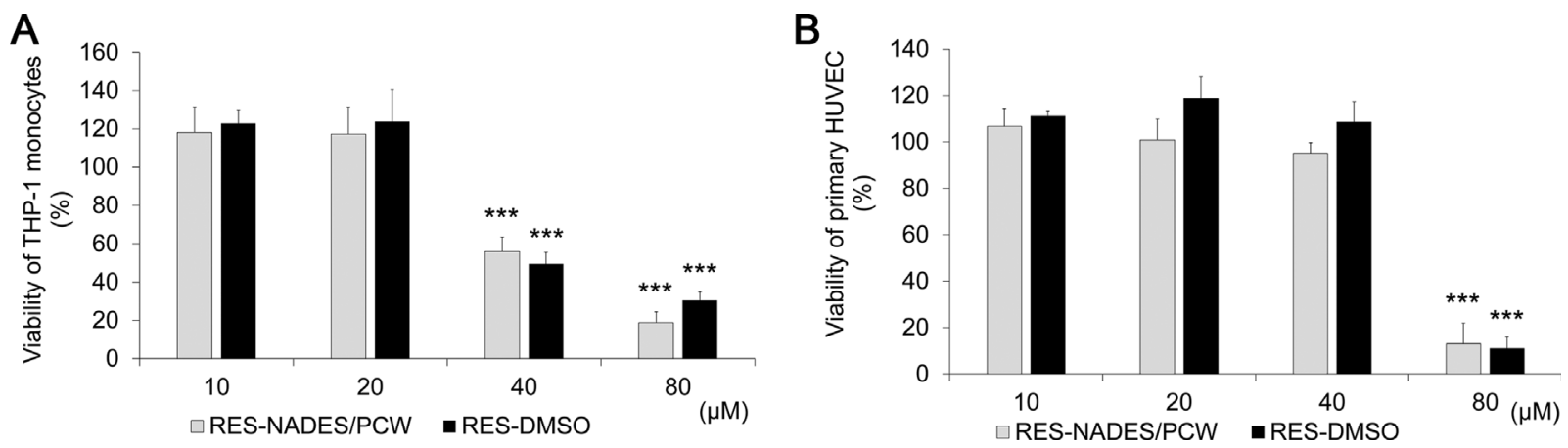

Figure 3. In vitro cytotoxic effects of resveratrol formulated in NADES/PCW or DMSO. NADES/PCW or DMSO concentrated at $0.2 \%$ was used to dissolve RES. In these Res solubilization conditions, increasing concentrations of $10,20,40$, and $80 \mu \mathrm{M} R \mathrm{RE}$ were submitted to MTT assay to assess their cytotoxic effects on either THP-1 monocytes (A) or primary HUVEC (B). Data were normalized against $0.2 \%$ of DMSO or NADES/PCW alone as negative control and 1\% SDS was used as positive control in cell culture medium. Values are mean of three experiments \pm standard deviation. ${ }^{* * *} p \leq 0.001$.

In contrast, at a final concentration of $0.5 \%$ of either NADES/PCW or DMSO vehicle alone allowed a high viability rate, close to $100 \%$, of tested cells. In contrast, RESNADES/PCW and RES-DMSO formulations exhibited similar toxic effects when used at 40 and $80 \mu \mathrm{M}$ on THP1 monocytes and HUVEC. Moreover, lethal concentrations able to kill $50 \%\left(\mathrm{LD}_{50}\right)$ of cultivated primary HUVEC were respectively reached at 60 and $64 \mu \mathrm{M}$ of RES-NADES/PCW and RES-DMSO respectively as compared with $\mathrm{LD}_{50}$ on THP-1 monocytes, which both were reached around $40 \mu \mathrm{M}$. These latter values showed that these formulations were less toxic for primary endothelial cells, which are the main physiological targets of the active MMP-9. In addition, independently of the RES formulation, below these concentrations, no toxic effects of RES were observed. These observations are extremely encouraging for the future in vivo experiments.

Since this RES-NADES/PCW was diluted in different cell culture media to proceed to test the biological activity of RES proposed in this work, we have observed that RES dissolved in the NADES/PCW vehicle keep its soluble form for longer durations in all these cultivation media in the absence or the presence of tested cell types as compared with DMSO. Therefore, the RES-NADES/PCW formulation, similarly to RES-DMSO formulation, is compatible with in vitro experiments to evaluate the RES capacity to inhibit

Table 3. LC50 effect of NADES/PCW- or DMSO-formulated RES on HUVEC and THP-1

$\mathrm{LC}_{50}(\mu \mathrm{M})$

\begin{tabular}{lcc} 
Cells & NADES/PCW & DMSO \\
\hline HUVEC & 60 & 65 \\
THP-1 & 40 & 40 \\
\hline
\end{tabular}

active MMP-9 produced by activated monocytes. Using a range of decreasing RES concentrations prepared in both formulations, we have observed that the NADES/PCW as compared with DMSO, resulted in a considerable enhancement by a factor of at least 10-fold of the RES capacity to inhibit the MMP-9 activity. Thus, when using RES a concentration as low as $1 \mu \mathrm{M}$ with the NADES/PCW formulation, RES was able to reduce MMP-9 activity up to $52 \%$ as compared with RES formulated with DMSO, which did not reduce this enzymatic activity at all, at this low concentration. These findings contribute to a considerable enhancement of the RES potential therapeutic effects in the context of NADES formulation, since they support that RES could exhibit its optimal effects under hormetic conditions. While, a similar reduction rate of MMP-9 activity induced by RES formulated with DMSO was only observed at concentrations of RES comprised between 10 and $20 \mu \mathrm{M}$.

Due to the poor bioavailability of RES, Sinclair et al. have suggested that a normal human weighing $75 \mathrm{~kg}$ would need a daily RES dose close to $100 \mathrm{mg} / \mathrm{kg} /$ day [40], which, obviously, is not applicable. Consequently, new derivatives and/or formulations of RES remain as unmet need that must be overcome to reach both acceptable bioavailability and activity of this compound.

This comparative study for the first time the possibility of getting improved drug's responses at low concentrations, as compared with traditional DMSO assays. We hypothesized that with the NADES formulation, these interesting RESbiological effects were probably due to the fact that NADES/ PCW could improve RES bioavailability, diffusion, transport, and cell uptake for specific cell compartment localization. In addition, taking into account the considerable number of possible NADES combinations, potentially it would be likely to optimize a particular NADES solvent for RES and any other drug compound for their clinical use at the lowest possible dosage. Since the limitation of the use of 
A
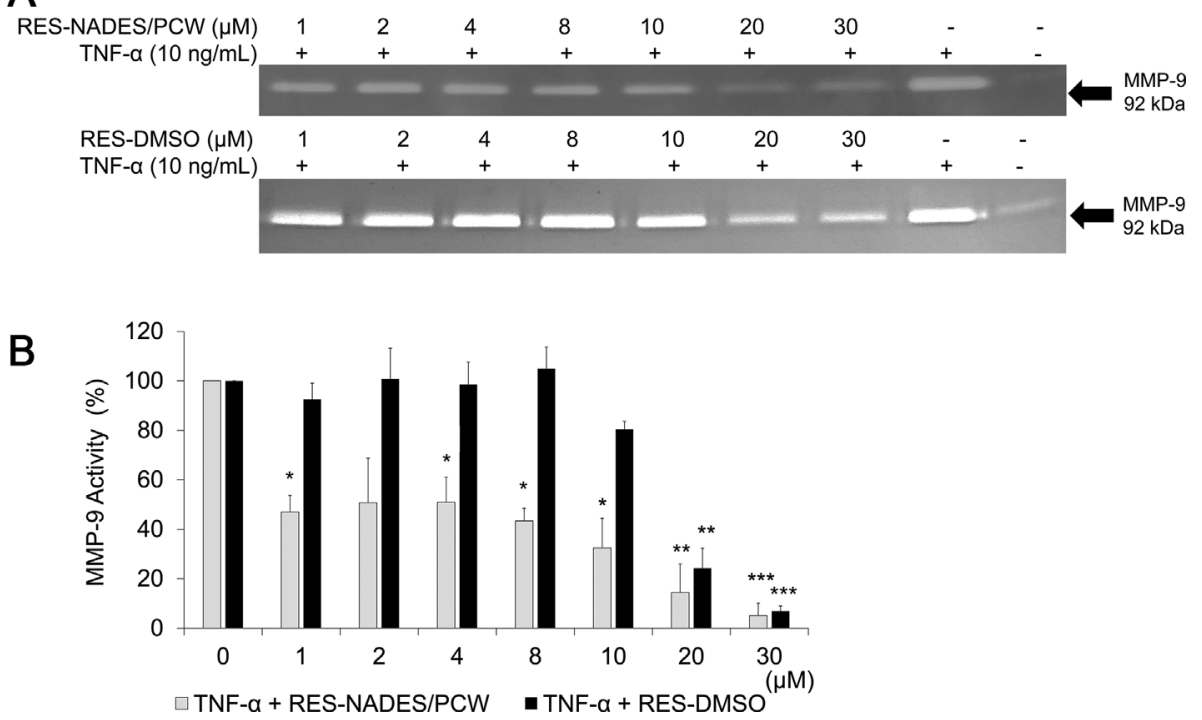

Figure 4. MMP-9-inhibitory effect of RES formulated in NADES/PCW or DMSO. The gelatinolytic activity of MMP-9 was revealed by a gel zymography assay. This assay evidenced that RES exhibit an inhibitory effect on the enzymatic activity of MMP-9 (A). In addition, RES dissolved in NADES/PCW exhibited a higher inhibitory activity of MMP-9 than the RES-DMSO formulation. Data from the pixel image analysis of individual zymography gel band of MMP-9 activity was plotted for each RES concentration (B). Data were normalized against negative control treated with $0.2 \%$ NADES/PCW or $0.2 \%$ DMSO in RPMI medium. Values are mean of three experiments \pm standard deviation. ${ }^{*} p \leq 0.05,{ }^{* *} p \leq 0.01$, and ${ }^{* * *} p \leq 0.001$.

DMSO in animal studies is due to its toxicity [41] and although very encouraging results of our work, further investigations (mainly pharmacokinetic properties) are required to set up in vivo assays of RES administration with non-covalent NADES formulations.

\section{Conclusions}

Altogether these results show, for the first time, that without altering the cell viability, the formulation of RES dissolved in the particular NADES/PCW solution significantly enhances the RES inhibitory effects on MMP-9 activity of a more than 10 -fold factor as compared with its formulation in DMSO. These data evidenced that NADES/PCW is able to enhance the hormetic mode of action of RES to decrease the MMP-9 activity. In addition, NADES are low-cost, simple to prepare, and biodegradable resulting in less toxic "green" compounds. Consequently, we propose that NADES could be use as formulation method that would be considered as a suitable "adjuvant" for druggable compounds, providing major advantages, including bio-compatibility, lowered toxicity, solubility, as well as the optimization of their active concentrations for an enhanced biological activity. Therefore, these findings could help to improve the pharmacological activity of RES for preclinical and clinical studies.
This work has been funded by participation of the IRD, the CNRS, the CIRAD, France. AS has been a PhD fellow at IRD supported by Misr University for Science and Technology (MUST), Egypt and IRD.

The authors have declared no conflict of interest.

\section{References}

[1] Brasnyo, P., Molnar, G. A., Mohas, M., Marko, L., et al., Resveratrol improves insulin sensitivity, reduces oxidative stress and activates the Akt pathway in type 2 diabetic patients. Br. F. Nutr. 2011, 106, 383-389.

[2] Magyar, K., Halmosi, R., Palfi, A., Feher, G., et al., Cardioprotection by resveratrol: A human clinical trial in patients with stable coronary artery disease. Clin. Hemorheol. Microcirc. 2012, 50, 179-187.

[3] Leighton, F., Cuevas, A., Guasch, V., Perez, D. D., et al., Plasma polyphenols and antioxidants, oxidative DNA damage and endothelial function in a diet and wine intervention study in humans. Drugs Exp. Clin. Res. 1999, 25, 133-141.

[4] Park, S. Y., Chae, S. Y., Park, J. O., Lee, K. J., Park, G., Gold-conjugated resveratrol nanoparticles attenuate the invasion and MMP-9 and COX-2 expression in breast cancer cells. Oncol. Rep. 2016, 35, 3248-3256.

[5] Misse, D., Esteve, P. O., Renneboog, B., Vidal, M., et al., HIV-1 glycoprotein 120 induces the MMP-9 cytopathogenic factor production that is abolished by inhibition of the p38 
mitogen-activated protein kinase signaling pathway. Blood 2001, 98, 541-547.

[6] Luplertlop, N., Misse, D., Bray, D., Deleuze, V., et al., Dengue-virus-infected dendritic cells trigger vascular leakage through metalloproteinase overproduction. EMBO Rep. 2006, 7, 1176-1181.

[7] Marsac, D., Garcia, S., Fournet, A., Aguirre, A., et al., Infection of human monocyte-derived dendritic cells by ANDES Hantavirus enhances pro-inflammatory state, the secretion of active MMP-9 and indirectly enhances endothelial permeability. Virol. f. 2011, 8, 223.

[8] Scherer, R. L., McIntyre, J. O., Matrisian, L. M., Imaging matrix metalloproteinases in cancer. Cancer Metastasis Rev. 2008, 27, 679-690.

[9] Zhang, Y., McCluskey, K., Fujii, K., Wahl, L. M., Differential regulation of monocyte matrix metalloproteinase and TIMP-1 production by TNF-alpha, granulocytemacrophage CSF, and IL-1 beta through prostaglandindependent and -independent mechanisms. F. Immunol. 1998, 161, 3071-3076.

[10] Bansal, S. S., Goel, M., Aqil, F., Vadhanam, M. V., Gupta, R. C., Advanced drug delivery systems of curcumin for cancer chemoprevention. Cancer Prev. Res. (Phila) 2011, 4, 1158-1171.

[11] Ponzo, V., Soldati, L., Bo, S., Resveratrol: A supplementation for men or for mice? f. Transl. Med. 2014, 12, 158.

[12] Pujara, N., Jambhrunkar, S., Wong, K. Y., McGuckin, M., Popat, A., Enhanced colloidal stability, solubility and rapid dissolution of resveratrol by nanocomplexation with soy protein isolate. F. Colloid Interface Sci. 2017, 488, 303-308.

[13] Visioli, F., The resveratrol fiasco. Pharmacol. Res. 2014, 90, 87.

[14] Cho, S. J., Jung, U. J., Choi, M. S., Differential effects of low-dose resveratrol on adiposity and hepatic steatosis in diet-induced obese mice. Br. F. Nutr. 2012, 108, 2166-2175.

[15] Timmers, S., Konings, E., Bilet, L., Houtkooper, R. H., et al., Calorie restriction-like effects of 30 days of resveratrol supplementation on energy metabolism and metabolic profile in obese humans. Cell Metab. 2011, 14, 612-622.

[16] Heeboll, S., Thomsen, K. L., Clouston, A., Sundelin, E. I., et al., Effect of resveratrol on experimental non-alcoholic steatohepatitis. Pharmacol. Res. 2015, 95-96, 34-41.

[17] Poulsen, M. M., Vestergaard, P. F., Clasen, B. F., Radko, Y., et al., High-dose resveratrol supplementation in obese men: an investigator-initiated, randomized, placebocontrolled clinical trial of substrate metabolism, insulin sensitivity, and body composition. Diabetes 2013, 62, 1186-1195.

[18] Calabrese, E. J., Converging concepts: Adaptive response, preconditioning, and the Yerkes-Dodson Law are manifestations of hormesis. Ageing Res. Rev. 2008, 7, 8-20.

[19] Calabrese, E. J., Resveratrol: An assessment of its dose response an introduction. Hum. Exp. Toxicol. 2010, 29, 977-979.

[20] Calabrese, E. J., Mattson, M. P., Calabrese, V., Dose response biology: The case of resveratrol. Hum. Exp. Toxicol. 2010, 29, 1034-1037.

[21] Mattson, M. P., Dietary factors, hormesis and health. Ageing Res. Rev. 2008, 7, 43-48.
[22] Richer, S., Patel, S., Sockanathan, S., Ulanski, L. J., 2nd, et al., Resveratrol based oral nutritional supplement produces long-term beneficial effects on structure and visual function in human patients. Nutrients 2014, 6, 4404-4420.

[23] Scapagnini, G., Davinelli, S., Kaneko, T., Koverech, G., et al., Dose response biology of resveratrol in obesity. $\mathcal{F}$. Cell Commun. Signal. 2014, 8, 385-391.

[24] Mattson, M. P., Cheng, A., Neurohormetic phytochemicals: Low-dose toxins that induce adaptive neuronal stress responses. Trends Neurosci. 2006, 29, 632-639.

[25] Atanackovic, M., Posa, M., Heinle, H., Gojkovic-Bukarica, L., Cvejic, J., Solubilization of resveratrol in micellar solutions of different bile acids. Colloids Surf B Biointerfaces 2009, 72, 148-154.

[26] Zu, Y., Zhang, Y., Wang, W., Zhao, X., et al., Preparation and in vitro/in vivo evaluation of resveratrol-loaded carboxymethyl chitosan nanoparticles. Drug Deliv. 2016, 23, 981-991.

[27] Ramalingam, P., Ko, Y. T., Improved oral delivery of resveratrol from $\mathrm{N}$-trimethyl chitosan-g-palmitic acid surface-modified solid lipid nanoparticles. Colloids Surf $B$ Biointerfaces 2016, 139, 52-61.

[28] Soo, E., Thakur, S., Qu, Z., Jambhrunkar, S., et al., Enhancing delivery and cytotoxicity of resveratrol through a dual nanoencapsulation approach. F Colloid Interface Sci. 2016, 462, 368-374.

[29] Summerlin, N., Soo, E., Thakur, S., Qu, Z., et al., Resveratrol nanoformulations: Challenges and opportunities. Int. F. Pharm. 2015, 479, 282-290.

[30] Choi, Y. H., van Spronsen, J., Dai, Y., Verberne, M., et al., Are natural deep eutectic solvents the missing link in understanding cellular metabolism and physiology? Plant Physiol. 2011, 156, 1701-1705.

[31] Durand, E., Lecomte, J., Baréa, B., Piombo, G., et al., Evaluation of deep eutectic solvent-water binary mixtures for lipase-catalyzed lipophilization of phenolic acids. Green Chem. 2013, 15, 2275.

[32] Weiz, G., Braun, L., Lopez, R., de María, P. D., Breccia, J. D., Enzymatic deglycosylation of flavonoids in deep eutectic solvents - aqueous mixtures: Paving the way for sustainable flavonoid chemistry. F. Mol. Catal. B Enzym. 2016, 130, 70-73.

[33] Dai, Y., van Spronsen, J., Witkamp, G. J., Verpoorte, R., Choi, Y. H., Natural deep eutectic solvents as new potential media for green technology. Anal. Chim. Acta. 2013, 766, 61-68.

[34] Mamajanov, I., Engelhart, A. E., Bean, H. D., Hud, N. V., DNA and RNA in anhydrous media: Duplex, triplex, and G-quadruplex secondary structures in a deep eutectic solvent. Angew. Chem. Int. Ed. Engl. 2010, 49, 6310-6314.

[35] Delaunay, J. C., Castagnino, C., Cheze, C., Vercauteren, J., Preparative isolation of polyphenolic compounds from Vitis vinifera by centrifugal partition chromatography. F. Chromatogr. A 2002, 964, 123-128.

[36] Abbott, A. P., Boothby, D., Capper, G., Davies, D. L., Rasheed, R. K., Deep eutectic solvents formed between choline chloride and carboxylic acids: Versatile alternatives to ionic liquids. F. Am. Chem. Soc. 2004, 126, 9142-9147. 
[37] Weksler, B. B., Subileau, E. A., Perriere, N., Charneau, P., et al., Blood-brain barrier-specific properties of a human adult brain endothelial cell line. FASEB f. 2005, 19, 1872-1874.

[38] Pan, D., Hill, A. P., Kashou, A., Wilson, K. A., Tan-Wilson, A., Electrophoretic transfer protein zymography. Anal. Biochem. 2011, 411, 277-283.

[39] Mosmann, T., Rapid colorimetric assay for cellular growth and survival: Application to proliferation and cytotoxicity assays. F. Immunol. Methods. 1983, 65, 55-63.

[40] Baur, J. A., Sinclair, D. A., Therapeutic potential of resveratrol: The in vivo evidence. Nat. Rev. Drug Discov. 2006, 56, 493-506.

[41] Galvao, J., Davis, B., Tilley, M., Normando, E., et al., Unexpected low-dose toxicity of the universal solvent DMSO. FASEB f. 2014, 28, 1317-1330. 